\title{
Un faux mensonge contre un vrai ? Imagination et réalité dans Amoralische Kinderklapper (1969) de Barbara Frischmuth
}

Eine falsche Lïge zur Aufdeckung einer wahren? Phantasie und Wirklichkeit in Barbara Frischmuths Amoralische Kinderklapper (1969)

A false lie against a true one? Imagination and reality in Barbara Frischmuth's Amoralische Kinderklapper (1969)

\section{Hélène Barrière}

\section{OpenEdition}

Journals

Édition électronique

URL : http://journals.openedition.org/ceg/1809

DOI : $10.4000 /$ ceg. 1809

ISSN : 2605-8359

Éditeur

Presses Universitaires de Provence

Édition imprimée

Date de publication : 1 décembre 2014

Pagination : 195-210

ISSN : 0751-4239

Référence électronique

Hélène Barrière, « Un faux mensonge contre un vrai ? Imagination et réalité dans Amoralische Kinderklapper (1969) de Barbara Frischmuth », Cahiers d'Études Germaniques [En ligne], 67 | 2014, mis en ligne le 17 décembre 2017, consulté le 04 novembre 2020. URL : http://journals.openedition.org/ ceg/1809; DOI : https://doi.org/10.4000/ceg.1809 


\title{
Un faux mensonge contre un vrai ? Imagination et réalité dans Amoralische Kinderklapper (1969) de Barbara Frischmuth
}

\author{
Hélène BARRIÈRE
}

Aix-Marseille Université

\begin{abstract}
Cinq enfants de trois à six ans, Robert, Lydia, Paula, Melchior et Leo ; une nurse, Annemarie ; une auberge, son grand jardin et ses alentours boisés, quelque part en Autriche ; un couple d'hôteliers, absorbés par leurs affaires : tels sont le décor et les personnages d'Amoralische Kinderklapper (Hochet amoral) de Barbara Frischmuth. Préciser tant soit peu cette épure exige de recouper les rares indications dispersées dans les dix-neuf petits textes de prose qui composent le livre, sans que la moindre continuité narrative en ordonne la succession. L'on obtient deux fois deux frères et sœurs, plus un petit orphelin : Paula (alias « Poppa, die lange Python») et Leo, les enfants de la maison, hébergent leurs cousins Robert et Lydia (dits «Rhesus» et «Libby-Kuh») ainsi que leur ami Melchior (surnommé «Freund Mowglie »), qui, pareil au héros de Kipling, a perdu ses parents ${ }^{1}$. Le lecteur doit se contenter de ces données parcimonieuses. L'équation conserve beaucoup d'inconnues, comme si l'univers de ces enfants devait se refuser à toute mise en formules.

Tel est bien, en effet, le propos de l'ouvrage. Sous cet angle, la question du mensonge revêt un tour particulier, qu'un premier extrait va nous permettre d'esquisser. Un après-midi, à la belle saison, Annemarie est chargée de retenir les enfants au jardin tandis que l'on prépare en secret une gâterie pour leur goûter. Elle a bien du mal à les occuper :
\end{abstract}

\footnotetext{
${ }^{1}$ À l'exception de Leo (mais son prénom contient déjà l'animal qui lui correspond et renvoie d'emblée au rôle de meneur de jeu dévolu à l'aîné de la petite troupe), le texte désigne toujours les enfants par leur surnom, sauf lorsqu'il rapporte les paroles qu'un adulte leur adresse. Singe (le Macacus rhesus est un macaque du nord de l'Inde), python et vache relèvent du même univers que Mowgli. En 1969, Le Livre de la jungle de Kipling (1894) jouit d'un regain de popularité : il a été porté à l'écran deux ans plus tôt par Walt Disney.
} 


\begin{abstract}
Ich versteh nicht, was ihr im Haus wollt, sagt Annemarie, wo es doch so schön ist hier draußen. Wenn ihr wollt, können wir Kirschen vom Baum pflücken Es gibt keine Kirschen mehr, sagt Freund Mowglie.

Die hat alle der Hagel gefressen, schreit Rhesus.

Du lügst wie gedruckt, sagt Libby-Kuh, der Hagel kann gar nicht fressen.

Du lügst, schreit Rhesus. Es heißt drücken. Ich kenn das Wort nur mit drücken.

Mit dir kann man nicht reden, sagt Libby-Kuh. ${ }^{2}$
\end{abstract}

Rhesus, le mangeur de cerises, se trahit en appliquant à la grêle le verbe qui désigne sa propre voracité ( fressen»). Libby-Kuh pointe le mensonge de Rhesus, mais le syllogisme qu'elle emploie est tronqué: entre la conclusion "Du lügst wie gedruckt » ${ }^{3}$ et la prémisse « der Hagel kann gar nicht fressen » (Libby-Kuh bouleverse au passage l'ordre logique), manque le maillon «Du bist's, der die Kirschen gefressen hat». Par le biais de cette omission s'établit dans l'énoncé une équivalence entre mentir et contrevenir à l'usage, ici lexical. Libby-Kuh a en outre recours à la tournure « lügen wie gedruckt», dont l'origine est ainsi définie par les dictionnaires : l'écriture manuscrite trahit l'auteur, tandis que l'anonymat des caractères d'imprimerie, joint au respect qu'ils inspirent, protège le coupable et encourage le mensonge ${ }^{4}$. Pour qu'un mensonge passe inaperçu et produise les effets escomptés, il faut que l'infraction au code se dissimule sous l'autorité de la chose établie, que la voix du menteur se fonde dans un consensus.

Libby-Kuh a donc déjà adopté, par mimétisme, la conviction dominante qui fait du mensonge un accroc au tissu du discours usuel ; mais pareil emploi de l'expression consacrée déroge à ce qui la fonde, puisque le mensonge incriminé est un échec. Son frère de trois ans, prenant à son tour modèle sur son aînée, dénonce comme "mensonge » l'entorse supposée à la formule toute faite, et commet, de même, une erreur de langage : «Du lügst, schreit Rhesus, es heißt drücken». Selon un mécanisme proche de la règle mathématique des signes ${ }^{5}$, ces deux conformismes conjugués, auxquels l'ironie du texte confère la forme d'une double impropriété, débouchent sur la

\footnotetext{
${ }^{2}$ Barbara FrISCHMUTH, Amoralische Kinderklapper, Frankfurt/ Main, Suhrkamp (st 224), 1975 [1 $1^{\text {re }}$ éd. Frankfurt/ Main, Suhrkamp, 1969], «Windspiele», p. 47. Par la suite, le texte sera toujours cité dans l'édition de 1975, sous la forme: AKK page. L'orthographe est celle de l'éditeur. Les petits textes qui constituent l'ouvrage ne sont pas numérotés, conformément à une logique de refus du déroulement narratif.

${ }^{3}$ La traduction française de cette expression ne saurait être littérale, elle exige le recours à une équivalence («Tu mens comme tu respires »). Une telle transposition rendrait toutefois impossible l'analyse qui suit. Pour des raisons semblables, nous nous verrons contrainte, dans l'ensemble de cet article, de fonder notre propos exclusivement sur le texte allemand.

${ }^{4}$ «Wer wie gedruckt lügt, der versteht es, seinen Lügen so gut den Schein der Wahrheit zu geben, dass man seinen Worten ebenso vertraut wie dem gedruckten Wort» (DUDEN 11, Redewendungen, Mannheim/ Leipzig..., Dudenverlag, 1992, p. 465). Ou encore : «Gemeint ist, dass die Handschrift ihren Urheber verrät, während die Druckerpresse anonym arbeitet : sie drückt auch die schlimmsten Lügen aus, und niemand kann sie dafür haftbar machen » (Heinz KüPPER, Wörterbuch der deutschen Umgangssprache, Stuttgart/ München..., Klett, 1997, p. 508).

5 «Moins par moins donne plus » : le produit de deux nombres affectés d'un signe moins est une valeur positive.
} 
mise en cause de la norme : «Es heißt drücken». Le nouveau dérapage lexical du petit Rhesus (du lügst wie gedrückt) fait basculer le mensonge du côté de la soumission à l'usage, imposée par la pression exercée sur l'individu dès le plus jeune âge. L'enfant contestataire est en effet privé de voix, au nom de son immaturité linguistique : « Mit dir kann man nicht reden », telle est la conclusion sans appel de Libby-Kuh, reprenant la stratégie des adultes.

Nous voici au cœur du questionnement qui se déploie dans Amoralische Kinderklapper. Temps de l'apprentissage linguistique et social, l'enfance est aussi le terrain privilégié du jeu. Elle n'est pas encore tout à fait réduite à appliquer des règles; elle parvient quelquefois à s'en saisir pour les faire jouer, elles aussi, et en desserrer l'étau. L'amoralité présente dans le titre renvoie non à la neutralité morale d'une nature opposée à la culture, mais à la force subversive d'une imagination qui fait fi des normes. L'intitulé choisi se réfère en effet au Moralische Kinderklapper (Hochet moral, 1794) de Karl August Musäus, plus connu pour ses Volksmärchen der Deutschen (17821786). Pour guider son lecteur sur cette piste, B. Frischmuth reprend en tête de son ouvrage l'exergue dont Musäus a pourvu le sien : " Mais les maximes morales les mieux venues sont pour la jeunesse nulles et non avenues $»^{6}$. Même si des travaux récents rectifient ce jugement ${ }^{7}$, Musäus possède la réputation d'un écrivain rationaliste qui n'accorde droit de cité au merveilleux que pour mieux le dissoudre dans la satire et l'ironie. À l'instar d'Eckart Herrmann $^{8}$, nous sommes donc en droit de supposer que l'amoralité dont B. Frischmuth défend la cause est celle d'une imagination qui refuse de se laisser dicter la loi de l'opinion commune.

Nous nous proposons de dégager les objectifs, les modalités et la portée de cette rébellion dont le pivot, comme nous l'avons entrevu, est la question du mensonge.

\footnotetext{
${ }^{6}$ " Jedoch die treflichen Moralen sind bey der Jugend Nullen ohne Zahlen ».

${ }^{7}$ Évelyne Jacquelin, par exemple, s'attache à revaloriser la place du merveilleux dans la littérature de l'Aufklärung et à montrer que le fantastique en émerge sans solution de continuité («Merveilleux et fantastique allemands», in Valérie TRITTER (dir.), Encyclopédie $d u$ fantastique, Paris, Ellipses, 2010, p.599-606, ici p. 599). Stefan Neuhaus observe que la stratégie narrative de Musäus ouvre la voie à E.T.A. Hoffmann et au Kunstmärchen (Märchen, Tübingen/ Basel, A. Francke Verlag, 2005, p. 78-88).

${ }^{8}$ «Wenn Musäus sich über die Naivität und Wundergläubigkeit der Märchenerzählung lustig machte, indem er das Erzählte ironisch auflöste und es verstandesmäßig erklärte, so bemüht sich Barbara Frischmuth gerade um das Gegenteil, nämlich um die Auflösung der kalten, sterilen, merkantilen Verstandeswelt der Erwachsenen » (Eckart HERRMANN, « Bücher für uns. Ein literarisch-musikalisches Magazin, zusammengestellt und präsentiert von Ekkehart Rudolph», Süddeutscher Rundfunk [Stuttgart], SF II, jeudi 5.2.1970, p. 8 du script de l'émission). Document consulté au Franz-Nabl-Institut für Literaturforschung der KarlFranzens-Universität Graz ( Schwarzbauer »-Zeitungsausschnittssammlung). Cet institut abrite le Vorlass (don du vivant de l'auteur) de B. Frischmuth (manuscrits, correspondance...).
} 


\section{Forum Stadtpark et le mensonge autrichien}

Le passage que nous venons de lire suffit à le prouver: Amoralische Kinderklapper n'appartient pas à la littérature de jeunesse, mais s'adresse aux adultes. B. Frischmuth, par ailleurs auteur d'une douzaine de livres pour enfants, s'en est expliquée très clairement. Dans cet ouvrage, elle a pour ambition de donner à l'enfant une consistance littéraire, en le libérant de son statut fictionnel le plus courant: celui, autobiographique, d'incarnation du passé de l'auteur ou celui de personnage secondaire ${ }^{9}$. Pour autant, offrir aux enfants une voix véritable ne signifie pas reproduire le contenu des échanges enfantins, mais épier les mécanismes qui gouvernent ceux-ci et les faire jouer sous les yeux des adultes, afin de mettre en lumière les potentialités que la socialisation émousse et de défendre la légitimité de ces pistes abandonnées. B. Frischmuth souligne que ses enfants sont des «constructions», des «personnages de langage ${ }^{10}$.

Un tel vocabulaire rend un son connu: il renvoie, bien sûr, à Ludwig Wittgenstein. Non tant au philosophe du Tractatus logico-philosophicus (1921) qu'à celui des Philosophische Untersuchungen (1953), qui comprend le langage en sa totalité comme un jeu, fragmenté en de multiples jeux de langage, et invente des «jeux de langage» fictifs auxquels il assigne un double objectif: éclairer, par comparaison, le fonctionnement du langage ordinaire, et nous permettre de «relativiser nos concepts, [de] [...] comprendre qu'ils ne sont ni les seuls possibles ni les meilleurs » ${ }^{11}$. L'on sait, en outre, que la critique de la conception augustinienne du langage qui forme le point de départ des Philosophische Untersuchungen se fonde pour partie sur les processus d'acquisition du langage par l'enfant ${ }^{12}$.

\footnotetext{
${ }^{9}$ «Ich habe in einigen meiner Bücher, die keine Kinderbücher sind, versucht, das Kind als literarischen Gegenstand zu emanzipieren. $\mathrm{Zu}$ emanzipieren von der rein autobiographischen Rolle (das Kind, das der Autor einmal war) und von der Anhängsel- und Statistenrolle, indem ich Kinder als literarisch gleichrangige Personen behandelte. In meinen Kinderbüchern [...] kommen oft gar keine Kinder vor. [...] Während in meinem [...] vor 20 Jahren erschienenen, nicht an Kinder adressierten Buch Amoralische Kinderklapper fast ausschließlich Kinder vorkommen, die auch gelegentlich aus Kinderbüchern zitieren » (Barbara FRISCHMUTH, « Lieb und ungestüm wie der Wind! », Die Welt, n 286, 7 décembre 1990, p. XIII, Archiv des FranzNabl-Instituts für Literaturforschung, S-Publ.7.4.1.1.1.2.5, Mappe «Primärtexte : über eigene Werke »).

${ }^{10} \ll[\mathrm{E}] \mathrm{s}$ ist halt wichtig, daß das Kind [...] nicht nur als erinnertes, sondern auch als Konstrukt vorkommt. [...] [I]n der Amoralischen Kinderklapper [habe ich] einmal versucht, die Kinder in ihrer Sprachwertung zu zeigen. Also erfundene Kinder, Kinder als Sprachfiguren» ("Gespräch mit Barbara Frischmuth », in Barbara FrISCHMUTH, Jahre. Zeit, Tschechow zu lesen. Unzeit. Bleiben lassen, textes choisis et présentés par Gisela ULRICH, Stuttgart, Klett, 1983, p. 108).

${ }^{11}$ Christiane CHAUVIRÉ, Jérôme SACKUR, Le vocabulaire de Wittgenstein, Paris, Ellipses, 2003 , p. 36

${ }_{12}$ Le $\S 6$, notamment, met en évidence par l'emploi du mot «Abrichtung» le lien entre apprentissage linguistique et conditionnement social (Ludwig WiTTGENSTEIN, Philosophische Untersuchungen, in Werkausgabe, vol. I, Tractatus logico-philosophicus, Tagebücher 19141916, Philosophische Untersuchungen, Frankfurt/ Main, Suhrkamp, 2006, p. 240).
} 
Des enfants, Wittgenstein en a eu beaucoup. À la fin des années soixante, sa postérité comprend une part non négligeable de l'avant-garde littéraire autrichienne, au sein de laquelle B. Frischmuth se trouve en bonne compagnie : à dix-neuf ans, en 1960, elle a participé à la fondation du Forum Stadtpark de Graz, aux côtés d'un Alfred Kolleritsch ou d'un Peter Handke. Forum Stadtpark tente de soulever la chape de plomb du conservatisme politique et culturel qui pèse sur les premières décennies de l'après-guerre. Au mépris de l'irréparable césure du nazisme, l'establishment proclame la pérennité de l'Autriche millénaire, héritière de l'Empire romain et du SaintEmpire romain germanique, dont l'aptitude supposée à concilier les contraires est appelée à faire référence dans l'édification européenne à venir. Cet avatar affadi de "l'idée d'Autriche» hofmannsthalienne ${ }^{13}$ fonctionne comme modèle identificatoire, ciment de la reconstruction nationale et pivot de l'activité touristique qui la soutient ${ }^{14}$. À la faveur du Traité d'État de 1955 dont le préambule exonère le pays de sa responsabilité dans la Seconde Guerre mondiale, responsables politiques et culturels s'emploient à effacer les traces des années brunes. Tout ensemble maquillage délibéré et "simple" silence, ce mensonge opère par action et par omission. Le terme de Vergangenheitsverdrängung dont on l'a désigné depuis suggère en outre la complexité d'un phénomène auquel concourent motivations conscientes et inconscientes, mensonge d'État et besoin individuel d'échapper à la culpabilité.

Les jeunes auteurs de Graz s'attaquent aux mécanismes profonds qui enrayent tout changement de paradigme politique et culturel. Ils pointent du doigt la «mythification », au sens barthésien, des structures existantes. "[L]e mythe », affirme Roland Barthes « est une parole choisie par l'histoire » ${ }^{15}$. Et de préciser :

\begin{abstract}
Ce que le monde fournit au mythe, c'est un réel historique, défini, si loin qu'il faille remonter, par la façon dont les hommes l'ont produit ou utilisé ; et ce que le mythe restitue, c'est une image naturelle de ce réel. [...] Le monde entre dans le langage comme un rapport dialectique d'activités, d'actes humains : il sort du mythe comme un tableau harmonieux d'essences. Une prestidigitation s'est opérée, qui a retourné le réel, l'a vidé d'histoire et l'a rempli de nature $[\ldots]^{16}$
\end{abstract}

Les écrivains de Forum Stadtpark s'élèvent contre l'imposture d'une réalité donnée comme immuable, alors qu'elle n'est, selon eux, qu'une

\footnotetext{
${ }^{13}$ Cf. Hugo von Hofmannsthal, «Die Idee Europa» (1917) et «Die österreichische Idee » (1917), in Gesammelte Werke, vol. II, Reden und Aufsätze II 1914-1924, Frankfurt/ Main, Fischer, 1979, p. $43-54$ et p. 454-458.

${ }^{14}$ Cf. Albert Berger, "Zur Funktion des Begriffs der "österreichischen Literatur" ", in Sigurd Paul SCHEICHL, Gerald STIEG (dir.), Österreichische Literatur des 20. Jahrhunderts. Französische und österreichische Beiträge, Innsbruck, Institut für Germanistik der Universität Innsbruck, 1986, p. 25-40.

15 Roland BARTHES, «Le mythe, aujourd'hui», in Mythologies, Paris, Seuil (« Points », 10), 1970 [1 $1^{\text {re }}$ éd. 1957], p. 194.

${ }^{16}$ Ibid., p. 230 (c'est Barthes qui souligne).
} 
construction langagière congruente à une forme de société qui entend se perpétuer à travers elle. Pour dénoncer ce mensonge, ils s'en prennent au fonctionnement usuel du langage. Imposé à l'enfant dès l'âge le plus tendre, le carcan des formules toutes faites l'empêche de penser ses expériences de façon autonome et le contraint à rester dans le rang :

\begin{abstract}
Unhintergehbar ist $[\ldots]$ den «Grazer» Autoren [...] die Erfahrung, daß experimentelle Einstellung zur Sprache eingefahrene, verordnete Weisen der Wirklichkeitsaneignung und Wirklichkeitsinterpretation, Absolutheitsansprüche geschlossener Ideologien, naiven Sprachrealismus zu unterlaufen vermag und daß diese Art von Subversivität eine eigene, wenn auch sehr vermittelte Form öffentlichen, ja politischen Handelns darstellt. ${ }^{17}$
\end{abstract}

L'un des paradoxes de cette bataille qui se veut avant-gardiste est qu'elle s'inscrit dans toute une tradition. Celle qui va de Kraus et Wittgenstein (le premier ayant influencé le second ${ }^{18}$ ) au Groupe de Vienne, en passant par les avant-gardes historiques telles l'expressionnisme, le dadaïsme ou le surréalisme. Sans parler du "père fondateur", Nietzsche, pour qui le langage est une convention rendue nécessaire par l'instinct grégaire de l'homme, la vérité une conformité à la désignation convenue des choses, et le mensonge un détournement des codes :

[W]eil [...] der Mensch zugleich aus Noth und Langeweile gesellschaftlich und heerdenweise existieren will, braucht er einen Friedensschluss und trachtet darnach dass wenigstens das allergröbste bellum omnium contra omnes aus seiner Welt verschwinde. [...] Jetzt wird [...] das fixirt, was von nun an „Wahrheit“ sein soll d. h. es wird eine gleichmässig gültige und verbindliche Bezeichnung der Dinge erfunden und die Gesetzgebung der Sprache giebt auch die ersten Gesetze der Wahrheit. [...] [Der Lügner] missbraucht die festen Conventionen durch beliebige Vertauschungen oder gar Umkehrungen der Namen. ${ }^{19}$

Nous n'entrerons pas ici dans les débats menés par la critique sur la spécificité autrichienne que constitue cette avant-garde littéraire tardive ${ }^{20}-$ si l'on peut oser l'oxymore - ou sur la légitimité de l'étiquette avant-gardiste revendiquée par Forum Stadtpark ${ }^{21}$, qui subit l'influence décisive du Groupe

\footnotetext{
${ }^{17}$ Kurt BARTSCH, «Damals vor Graz: Die verspätete Aneignung von Moderne und Avantgarde in der Literatur aus dem Umkreis von Forum Stadtpark Graz und der Zeitschrift manuskripte », Études Germaniques, 50 ${ }^{\mathrm{e}}$ année, n 2, avril-juin 1995, p. 300.

${ }^{18}$ Cf. Allan JANIK, «Kraus, Wittgenstein et la philosophie du langage », Austriaca, n ${ }^{\circ} 49$, décembre 1999, p. 67-83.

${ }^{19}$ Friedrich NIETZSCHE, « Ueber Wahrheit und Lüge im aussermoralischen Sinne », in Die Geburt der Tragödie. Unzeitgemäße Betrachtungen I-IV. Nachgelassene Schriften 1870-1873, éd. par Giorgio COLLI et Mazzimo MONTINARI, München, dtv, 1999, p. 877-878.

${ }^{20}$ Cf. Klaus KASTBERGER, «Wien 50/60. Eine Art einzige österreichische Avantgarde », in Thomas Eder, Klaus KastBerger (dir.), Schluß mit dem Abendland! Der lange Atem der österreichischen Avantgarde, Wien, Zsolnay (« Profile », 5), 2000, p. 5-7.

${ }^{21}$ Cf. Gunter FALK, Alfred KOLLERITSCH, «marginalie», manuskripte, $4^{\mathrm{e}}$ année, $\mathrm{n}^{\circ} 12$, 1964, p. 1 (cité dans Kurt BARTSCH, «Das Forum Stadtpark Graz und seine Zeitschrift
} 
de Vienne né quelque dix ans avant lui ${ }^{22}$. Qu'il nous suffise de mentionner les fructueux échanges entre les jeunes écrivains de Graz et leurs aînés viennois : les premiers offrent aux seconds, dans leur revue manuskripte, une tribune qui leur permet de sortir de la quasi-confidentialité ; les seconds font profiter leurs cadets des pistes ouvertes par une rigueur programmatique et une radicalité littéraire qui font défaut à Forum Stadtpark. Ainsi, certains aspects d'Amoralische Kinderklapper ne sont pas sans rappeler la démarche de Hans Carl Artmann, l'une des figures de proue de l'avant-garde viennoise, dont l'écriture foisonnante et protéiforme échappe toutefois assez vite aux contraintes de groupe. C'est, du reste, le fils d'Artmann qui dessine la couverture de la première édition de l'ouvrage $\mathrm{e}^{23}$. Peu encline, tout comme Artmann, au positionnement théorique, B. Frischmuth baigne toutefois dans cette effervescence littéraire, dont les préoccupations anticipent sur certains points le combat anti-autoritaire mené par les étudiants à la fin des années soixante. C'est en 1967 que la jeune femme, qui a déjà publié quelques textes dans manuskripte, fait ses véritables débuts: elle effectue une lecture remarquée du récit Meine Großmutter und ich lors de l'une des dernières réunions du Groupe 47. Un an plus tard paraît Die Klosterschule, qui la révèle au grand public. Dans le premier texte, B. Frischmuth dénonce le brouillage psychique que provoquent, chez l'adolescent, des principes éducatifs fondés sur un catholicisme à la fois férocement coercitif et d'une sensiblerie exacerbée; dans le second, elle fustige la soumission aux structures patriarcales inculquée dans les internats catholiques de filles.

Les jeunes enfants d'Amoralische Kinderklapper viennent compléter et élargir cette critique. L'ouvrage s'attaque cette fois au "formatage" social de la petite enfance et se penche sur des mécanismes plus généraux, moins étroitement liés à l'idéologie religieuse. Les armes de la dénonciation diffèrent. Die Klosterschule assemble à joints vifs des bribes de quotidien et des fragments de discours éducatif. Cette absence de liant narratif met à nu le mensonge d'un tout immuable et harmonieux, idéologie religieuse, politique et sociale autant qu'esthétique. Cependant, citation et montage condamnent l'écriture à ne pouvoir se dégager, dans le fond comme dans la forme, de ce

manuskripte in den 1960er Jahren. Eine Avant-Garde ?», in Kurt BARTSCH, Gerhard MELZER (dir.), Trans-Garde. Die Literatur der "Grazer Gruppe ». Forum Stadtpark und " manuskripte », Graz, Droschl, 1990, p. 12).

${ }^{22}$ Certains, tel Reinhard Priessnitz, font ainsi grief aux premières productions des jeunes écrivains de Graz de n'être que " post-expérimentales ", c'est-à-dire synthèse commercialement réussie entre expérimentation langagière calquée sur celle du Groupe de Vienne et modes de narration conventionnels (Reinhard PRIESSNITZ, Mechthild RAUSCH, « tribut an die tradition. aspekte einer postexperimentellen literatur », in Peter LAEMMLE, Jörg DREWS (dir.), Wie die Grazer auszogen, die Literatur zu erobern. Texte, Porträts, Analysen und Dokumente junger österreichischer Autoren, München, dtv, 1979, p. 126-152).

${ }^{23}$ Cf. Reinhard URBACH, «AMORALISCHE KINDERKLAPPER », Wort und Wahrheit, n 25 , 1970, p. 89 (Franz-Nabl-Institut für Literaturforschung, « Schwarzbauer »-Zeitungsausschnittssammlung). 
qu'elle stigmatise ${ }^{24}$. Amoralische Kinderklapper défend, comme nous l'avons $\mathrm{vu}$, les droits d'une imagination aux potentialités sinon intactes, du moins à peine altérées par l'éducation. Instrument de dénonciation, l'imagination est aussi outil de création. Non content de mettre au jour le mensonge d'un usage linguistique qui se donne pour LA vérité, le texte s'emploie à dessiner des constellations expressives inédites qui sont autant de mondes possibles.

\section{Contre le mensonge de l'usage : le chatoiement fantastique du langage}

Ces "débordements de langage" prolongent le rôle de trublion séculaire joué par l'imagination. Depuis l'Antiquité, la « folle du logis» constitue une menace pour la codification du discours. La rhétorique l'a placée au cœur des débats sur le possible et l'impossible, l'admis et l'interdit, le vrai et le faux; elle l'a consignée dans les limites du raisonnable et du vraisemblable, plus ou moins étroites selon les époques. Ces discussions, trop vastes pour être retracées ici, ont été abondamment et excellemment étudiées, notamment par Renate Lachmann ${ }^{25}$. C'est au tournant du XIX ${ }^{\mathrm{e}}$ siècle, observe-t-elle, que l'imagination s'affranchit assez de ce qui la bride pour donner naissance à un nouveau mode d'écriture, le fantastique. Cependant, même alors, elle ne peut se déprendre entièrement des codes de la représentation mimétique. Ils lui demeurent nécessaires pour s'en démarquer. Ainsi le fantastique apparaît-il à R. Lachmann comme une intensification du régime fictionnel, poussé au-delà de ses limites - comme une hérésie :

[D]as Reale [kann] als die Präsenz einer funktionierenden Kultur [...] interpretiert werden [...]. Das Phantastische [...] kann trotz seiner Aufkündigung der fiktionalen Darstellungsregeln und der versuchten Desintegration des Bestehenden nicht ohne die Welt des Realen, Möglichen, Rationalen bestehen. ${ }^{26}$

Vielleicht läßt sich sagen, daß die Phantastik als literarische Häresie auftritt, indem sie mit den Regeln spielt, die eine Kultur für ihren Fiktionsdiskurs geltend macht. In der Entstellung der Kategorien von Zeit, Raum und Kausalität überschreitet die Phantastik die Normen der mimetischen Konvention [...]. Das Phantastische erscheint als eine Art "verschärfter Fiktion". 27

\footnotetext{
${ }^{24} \mathrm{Il}$ en est de même pour beaucoup d'écrivains de Forum Stadtpark à leurs débuts. On en a fait notamment reproche à Alfred Kolleritsch en sa qualité d'éditeur de la revue manuskripte: «Elisabeth Wiesmayr [...] kritisiert, [...] [n] egativ sei Kolleritsch [...] selbst den Positionen verhaftet, die er anprangert » (BARTSCH, «Damals vor Graz », p. 293-294).

25 Renate LachmanN, Erzählte Phantastik. Zur Phantasiegeschichte und Semantik phantastischer Texte, Frankfurt/ Main, Suhrkamp, 2002.

${ }^{26}$ Ibid., p. 10.

${ }^{27}$ Renate LachmanN, «E.T.A. Hoffmanns Phantastikbegriff », in Gerhard NeUmanN (dir.), "Hoffmanneske Geschichte". Zu einer Literaturwissenschaft als Kulturwissenschaft, Würzburg, Königshausen \& Neumann, 2005, p. 140.
} 
Dans Amoralische Kinderklapper, l' « hérésie » fantastique n'opère pas au niveau de la fiction, mais s'installe à l'intérieur même du langage. Certes, là n'est pas l'originalité de B. Frischmuth :

Innerhalb einer weit gefaßten Konzeption hat jede Irrealisierung von Sprache ein phantastisches Moment, wie es etwa für die « kühne Metapher », generell für die sogenannte dunkle Lyrik und jedes sprachliche Erzeugnis einer nicht realitätsbezogenen Einbildungskraft gilt.

note R. Lachmann ${ }^{28}$. Ce qui fait l'intérêt du texte, c'est la mise en bouches enfantines de ce langage fantastique: elle redouble l'offensive de l'imagination contre la mise en mots convenue du "réel"" .

Nous voudrions à présent observer comment cet objectif est mis en œuvre. Afin de respecter le cadre imparti à cet article, nous nous limiterons à un petit nombre d'exemples. Il n'épuise pas toutes les pistes que l'ouvrage de B. Frischmuth ouvre à la réflexion sur le mensonge.

\section{Monstres lexicaux}

Le mode de distanciation intralinguistique qui rend le discours usuel « infamilier », pour emprunter à Philippe Forget le néologisme par lequel il traduit l'adjectif « unheimlich ${ }^{30}$, prend la forme de monstres lexicaux. Ainsi dans le chapitre « Als die Wünsche noch an den Bäumen hingen », où LibbyKuh et Freund Mowglie confrontent leurs goûts culinaires :

Magst du denn Blutwurst? fragt Libby-Kuh.

Es geht, sagt Freund Mowglie, aber lieber esse ich Hornochsenfilet [...] und dazu ein Glas Essigundöl mit einem Eiszeitwürfel zu fünfzig Millionen.

${ }^{28}$ LACHMAnN, Erzählte Phantastik, p. 13. Cf. aussi Christine LubKoll, Inge STEUTZGER, «Einleitung », in Christine LubKoll (dir.), Das Imaginäre des Fin de siècle. Ein Symposium für Gerhard Neumann, Freiburg im Breisgau, Rombach, 2002, p. 8 : «In der Literatur wird das Imaginäre vor allem über phantasmatische Bildwelten entbunden, aber auch über [...] Experimente mit der Materialität der Sprache: Klang, Rhythmus, anti-mimetische Ausdruckskunst ».

${ }^{29}$ L'adjectif « fantastique » apparaît sous la plume de certains critiques, mais sans mise en perspective historique ni justification plus poussée : «Erstaunlich ist die Sprachphantasie dieser jungen Autorin, erstaunlicher noch die Sicherheit, mit der sie im ganzen Buch den Sprechton der Kinder beherrscht und festhält : herb und phantastisch zugleich, hart an der Realität und dann wieder die Realität überspringend» écrit par exemple Elsbeth Pulver («Barbara Frischmuth : Amoralische Kinderklapper », Schweizer Monatshefte [Zürich], 49 année, $\mathrm{n}^{\circ} 9$, décembre 1969, Franz-Nabl-Institut für Literaturforschung, "Schwarzbauer »-Zeitungsausschnittssammlung).

30 Philippe FORGET, «Présentation: Hoffmann l'infamilier», in E.T.A. HofFMANN, Tableaux nocturnes, vol. I, Paris, Imprimerie nationale, 1999, p. 47. Le terme est généralement rendu par «étrangement inquiétant», depuis la traduction par Marie Bonaparte de l'essai de Freud Das Unheimliche.

${ }^{31}$ AKK 30. 
Les menus concoctés par les enfants comportent, dans leur incongruité, une bonne dose de poétique surréaliste. Mais alors que celle-ci érige l'arbitraire en valeur absolue ${ }^{32}$, les recettes enfantines prennent pour cible, avec plus de méthode qu'il n'y parait, les repères du quotidien. Le texte lexicalise, par exemple, des partenariats culinaires consacrés par l'usage («Essigundöl»). Il fige ainsi en mariages des alliances occasionnelles, mettant à l'index le carcan de l'habitude. Ou, au contraire, il disloque un composé lexicalisé (Eiswürfel) en y enfonçant le coin d'un substantif nouveau (Zeit), qui entre à son tour en composition avec l'un des éléments initiaux (Eiszeit) et fait éclater les distinctions espace-temps, concret-abstrait («Eiszeitwürfel »). Ou bien encore il brouille les lois de la composition ellesmêmes, comme dans " Hornochsenfilet », où Ochse paraît osciller entre Horn et Filet, sans parvenir à trouver sa place dans le trio. Allié potentiel de l'un comme de l'autre, il est pourtant malvenu au milieu des deux : le composé ainsi formé fonctionne non comme un exhausteur de goût, mais de bêtise : le filet d'un corniaud (Hornochse) n'en est-il pas le meilleur morceau ? Éliminer Ochse s'avère tout aussi impossible (Hornfilet), sous peine d'un oxymore qui annihile l'opposition dur-tendre.

Dans cette altération des catégories du temps et de l'espace, cet anéantissement des distinctions logiques, cette irrésolution, l'on aura reconnu bien des éléments qui, de l'avis des spécialistes, caractérisent le fantastique ${ }^{33}$. Quant à l'oxymore, il est au cœur de l'une de ses définitions récentes :

Das Phantastische basiert auf einem Verfremdungsverfahren, das ein reguläres Realitätssystem durch ein zweites, wunderbares Realitätssystem in Frage stellt. [...] Das Phantastische als "kosmologisches Oxymoron" ist Konkurrenz und Negation. Es setzt sich aus Realitätssystemen zusammen, denen es sich gleichzeitig verweigert. $^{34}$

Principe de fonctionnement du fantastique, la contradiction est aussi, selon Harald Weinrich, celui de toute métaphore. Contrairement à l'opinion reçue, les métaphores les plus audacieuses ne sont pas, à ses yeux, celles qui possèdent l'«amplitude métaphorique » (Bildspanne) la plus grande, mais celles où comparant et comparé sont sémantiquement peu éloignés. Le « lait noir de l'aube $»^{35}$ de Paul Celan recèle d'après lui plus d'audace que les

\footnotetext{
32 « Pour moi, la plus forte [image surréaliste] est celle qui présente le degré d'arbitraire le plus élevé $[\ldots]$; celle qu'on met le plus longtemps à traduire en langage pratique » (André BRETON, Manifeste du surréalisme, Paris, Gallimard (« Idées », 23), 1977 [1 ${ }^{\mathrm{re}}$ éd. 1924], p. 52.

${ }^{33} \mathrm{Ce}$ constat est à présent assez consensuel. Nous n'évoquerons ici que l'étude de Tzvetan Todorov, parfois controversée mais à maints égards pionnière, et sa formule demeurée célèbre : «Le fantastique, c'est l'hésitation éprouvée par un être qui ne connaît que les lois naturelles, face à un événement surnaturel» (Introduction à la littérature fantastique, Paris, Seuil

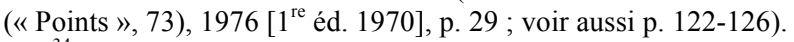

${ }^{34}$ Uwe DURST, Theorie der phantastischen Literatur, Münster, LIT Verlag, 2010, p. 116.

35 "Schwarze Milch der Frühe» (Paul CELAN, «Todesfuge», in Ausgewählte Gedichte, Frankfurt/ Main, Suhrkamp, 1979, p. 18).
} 
«parfums pourpres du soleil des pôles» de Rimbaud ${ }^{36}$, car la métaphore de Celan ne nous transporte pas en territoire inconnu, mais déroge simplement à la couleur d'un breuvage familier ${ }^{37}$.

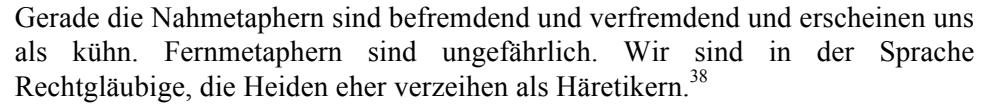

Les composés métaphoriques de $\mathrm{B}$. Frischmuth relèvent de cette hérésie impardonnable. Ils constituent de véritables «outils démiurgiques » ${ }^{39}$. Le contexte culinaire les ancre dans un quotidien que leurs pirouettes sémantiques redessinent à plaisir. Ils soumettent les références religieuses et culturelles à un traitement linguistique inédit qui les désacralise et les prive de leur fonction de repères. Libby-Kuh, en quête d'une boisson propre à accompagner son plat favori, reçoit de Mowglie le conseil : «Dazu paßt am besten leberfarbener Puschkinfäustesaft mit einem Drechsel-Löffel zum Umspiralen $^{40}$. " La Scène de Faust de Pouchkine (1828), cachée sous un pluriel qui dément l'unicité de l'œuvre d'art et lui confère la violence d'un coup de poing, se métamorphose en jus de fruit à la sombre couleur - version light de la vodka auquel le poète russe fut contraint de prêter son nom ${ }^{41}$. Libby-Kuh et Mowglie, que Poppa met ensuite au défi de deviner le cadeau d'anniversaire qu'elle désire, poursuivent leur recomposition du paysage culturel :

[D]iesmal wünsch ich mir [...] was[, sagt Poppa].

Was denn? fragt Libby-Kuh.

Etwas Extraartiges.

[...] [E]inen Rosmarien-Kranz oder einen Thymerjahn? fragt Freund Mowglie. $^{42}$

Rosenkranz, le courtisan qui trahit l'amitié de Hamlet et dont le nom signifie « rosaire ", s'ensauvage et revêt les senteurs provençales du romarin (Rosmarin) ou du serpolet (Marienkranz, appellation populaire du Quendel). Le Turnvater Jahn, symbole d'un nationalisme aux relents racistes et xénophobes fustigé par Heinrich Heine, voit son patronyme changé en prénom exotique (Erdjan) et fleurant bon le thym (Thymian) de la garrigue ${ }^{43}$.

\footnotetext{
36 Arthur RIMBAUD, «Métropolitain» (Illuminations), in Euvres, éd. par Suzanne BERNARD, Paris, Garnier, 1960, p. 291.

${ }^{37}$ Harald WeINRICH, "Semantik der kühnen Metapher » [1963], in Anselm HaVERKAMP (dir.), Theorie der Metapher, Darmstadt, Wissenschaftliche Buchgesellschaft, 1983, p. 325-328.

${ }^{38}$ Ibid., p. 328.

${ }^{39}$ « demiurgische Werkzeuge » (ibid., p. 331).

${ }^{40}$ AKK 30

41 «Puschkin» est depuis 1959 une marque de vodka dont la recette, importée en Allemagne par des émigrés fuyant la révolution russe, remonte aux années 1920.

${ }^{42}$ AKK 31.

${ }^{43}$ Nous tenons à remercier Ch. Januel, K. Wimmer, H. Berke et W. Fink d'avoir attiré notre attention sur la proximité entre Thymerjahn et Dummerjan (imbécile), dont l'équivalent
} 
Le rattachement à la sphère sensorielle des saveurs et des odeurs charge les repères culturels ainsi détrônés d'une vitalité qui les déploie dans des directions nouvelles.

\section{La lettre morte ressuscitée : propre et figuré}

Cette dynamique habite le texte de bien d'autres manières encore. À Poppa qui raille l'incapacité de Libby-Kuh et Mowglie à trouver ce qui lui ferait plaisir, ce dernier propose pour finir : « Oder eine Faß-Taube oder einen Pech-Vogel $[\ldots]^{44}$ ?»

La substitution d'une seule lettre à une autre dote l'inanimé d'une vie étrange, transformant une douve ${ }^{45}$ (Fassdaube) en pigeon (Taube). L'imagination enfantine insuffle vie à la lettre "morte" du sens abstrait: le «Pech-Vogel»", placé sur le même plan grammatical que "Faß-Taube», redevient oiseau. Le trait d'union permet en quelque sorte d'en visualiser l'envol libérateur hors de la cage du mot composé, figé dans son sens figuré.

T. Todorov, qui au cœur de la vague structuraliste du début des années soixante-dix est le premier à s'intéresser au fantastique comme discours, voit dans ce jeu sur le propre et le figuré l'un de ses ressorts : le fantastique «naît souvent de ce qu'on prend le sens figuré à la lettre ${ }^{47}$. Ainsi en va-t-il par exemple dans Vera de Villiers de l'Isle-Adam, où le récit réalise le sens propre de l'incipit "[1]'Amour est plus fort que la Mort», inspiré du Cantique des Cantiques ${ }^{4}$, et redonne vie à une femme éperdument aimée.

Le chapitre d'Amoralische Kinderklapper intitulé «Wie die Rede auf den Tod kommt» repose sur ce mécanisme. Les enfants, en révolte contre le programme de jeu imposé par Annemarie, se roulent dans l'herbe du jardin et la mordent à belles dents (« die Kinder [...] beißen ins Gras » $\left.{ }^{49}\right)$. "Mourir" les place hors d'atteinte, tout en leur laissant le loisir de tourmenter d'une façon ou d'une autre les "vivants". Mais tel est pris qui croyait prendre: Annemarie, laissant tomber sur eux quelques poignées de terre, leur donne une sépulture. Les voici condamnés à trouver le calme et le repos - éternel de préférence ! Se voyant devinés, ils parviennent à lui faire enjamber une vieille

autrichien est Dummian. Cette parenté, qui nous avait échappé, ne contredit cependant pas l'interprétation que nous proposons, ainsi que l'indiquent le choix du terme allemand et le $h$ de "Thymerja $h \mathrm{n}$ ».

${ }^{44}$ AKK 31.

${ }^{45}$ Planche servant à la fabrication des tonneaux.

${ }^{46}$ Le terme «Pechvogel» désigne un être malchanceux. La formule «avoir la poisse », à laquelle ne correspond aucun substantif français, relève de la même image que l'allemand «Pech haben ».

${ }^{47}$ TODOROV, Introduction à la littérature fantastique, p. 82.

48 «Fort comme la Mort est Amour» (Ancien Testament, TOB, Paris, Éd. du Cerf, 1987, p. 1610).

49 AKK 8. L'équivalent français de cette figuration imagée de la mort est «mordre la poussière ». 
lame de rasoir dissimulée sous un livre et l'attirent ainsi dans la mort, lui assignant la condition subalterne dévolue à la moindre ancienneté :

Wir haben dich über die Klinge springen $\operatorname{lassen}^{50}$ [, sagt Leo,] und jetzt bist du genauso tot wie wir, nur daß dich noch niemand beerdigt hat. [...]

$[\mathrm{U}] \mathrm{nd}$ du hast überhaupt nichts zu reden, denn du bist noch lang nicht so lang tot wie wir. ${ }^{51}$

Une situation concrète (« ins Gras beißen », « über die Klinge springen ») s'est au fil du temps dématérialisée en figuration abstraite, euphémique, de la douleur mortelle. Pareille édulcoration tient la mort à distance; sa fécondité (leitmotiv des mythes, dans lesquels la mort accouche de la vie au rythme d'un temps circulaire) est bridée par ce processus. La remise en "jeu" d'expressions figées permet aux enfants de prendre possession de la mort, de la réintégrer à la vie.

\section{Faux-et "vrai"-semblant}

Abolir la frontière entre propre et figuré conduit, pour finir, à effacer celle qui sépare imagination et réalité. C'est ce qu'illustre la suite du chapitre, où, une fois "morts", les enfants trouvent le temps long :

Und jetzt spucken wir, sagt Rhesus, ja? und spuckt sich einen Spuckerling vor die eigenen Füße.

Das können wir erst um Mitternacht, fährt Leo ihn an.

Und was machen wir, bis es Mitternacht wird ? [...]

Ich weiß was [...]. Annemarie steht vom Boden auf und holt den Wecker von der Kommode. Es genügt doch, wenn wir den Zeiger nach vor richten.

Du mogelst, sagt Freund Mowglie, und Tote sollten sich wirklich schämen, so etwas zu tun.

Da räkelt sich Poppa, die lange Python, und sagt, ich weiß $[\ldots]$ was Besseres, wir spielen als ob.

Als ob was? fragen die anderen.

Hört her. Es ist als ob es schon finster wäre, als hätte es eben zwölf geschlagen und wir wären auferstanden.

Es ist aber nicht als ob, sagt Annemarie, wir wollen doch nur so tun als ob.

Wenn wir so tun als ob, dann ist es als ob, sagt Leo $[\ldots] .^{52}$

Chez Leo, l'aîné des petits compagnons, l'assimilation des codes de représentation usuels (mimétiques et non mimétiques) est plus avancée que chez Rhesus, de trois ans son cadet. Contrairement à ce dernier, il entend respecter la tradition qui fait de minuit l'heure où s'éveillent les fantômes. Mais Leo n'a pas encore renié la cause de l'imagination. Il prend la logique commune à son propre piège : le « tun» du « tun als ob», restauré dans toute

\footnotetext{
${ }^{50} \mathrm{~L}$ 'expression française correspondante est « passer au fil de l'épée ».

${ }^{51}$ AKK 10-11.

${ }^{52}$ AKK 11
} 
son intensité de verbe d'action, ancre dans la réalité les virtualités envisagées : «Wenn wir so tun als ob, dann ist es als ob». Dans la bouche de Leo, s'inverse l'opération gouvernée d'ordinaire par «als ob»: au lieu de substituer du virtuel à du réel, il change le virtuel en réel. Ce faisant, Leo radicalise aussi l'usage habituel de cette locution conjonctive dans les récits fantastiques. T. Todorov range en effet « comme si » au nombre des formules modalisantes (" on eût dit », « il semblait que », etc.) destinées à favoriser la concurrence entre une interprétation des faits conforme à la représentation convenue du réel et une explication qui y contrevient ${ }^{53}$. Leo, lui, fait de l'imagination en action (le jeu) un territoire du réel extensible à l'infini. L'adulte se "prête" au jeu des enfants, mais ne se "donne" jamais: sa participation repose sur le faux $x$-semblant. En avançant le réveil, Annemarie se livre en effet à une manipulation superficielle (" Es genügt doch, wenn... »), qui s'exerce sans risques sur la figuration arbitraire d'une réalité impalpable, le temps. Mowglie dénonce ce respect de pure façade à la règle du jeu (« [d]u mogelst »), peu compatible avec la droiture que l'on suppose aux morts. Selon l'adage que Mowglie semble avoir déjà fait sien, la mort ne sonne-t-elle pas pour tout vivant l'heure de vérité ? En jouant à « comme si », l'enfant se réclame, pour sa part, de ce que l'on pourrait nommer le "vrai-semblant", opposé à la vraisemblance: la seconde se définit, si l'on suit Gérard Genette, par la conformité à l'opinion commune, comprise comme «un corps de maximes et de préjugés qui constitue tout à la fois une vision du monde et un système de valeurs ${ }^{54}$; le premier comme l'exploration concrète des pistes ouvertes par l'imagination.

\section{Raconter des histoires}

Les enfants d'Amoralische Kinderklapper, campés comme les défenseurs d'une juste cause, ne sont-ils pas trop beaux pour être vrais - et même pour être inventés? Non pas : nous les avons pris en flagrant délit de mensonge, les avons vu peu à peu contaminés par le discours dominant. L'ouvrage les montre en outre, dans le chapitre « Der Leichenschmaus », se repaissant de corps dépecés, béants ou atrocement boursoufflés - détails macabres dont Poppa agrémente son récit de la mort violente de ses grands-parents. Dans le chapitre « Die Wildnis », ils sont des Indiens en chasse mettant à mort un chat baptisé pour l'occasion «bête sauvage» («wildes Tier»), à la grande indignation d'auditrices horrifiées ${ }^{55}$ qui alertent presse écrite et société protectrice des animaux ${ }^{56}$. Et les adultes, tous des menteurs ? Il conviendrait

\footnotetext{
${ }^{53}$ TODOROV, Introduction à la littérature fantastique, p. 85-86.

${ }^{54}$ Gérard GENETTE, «Vraisemblance et motivation », in Figures II, Paris, Seuil (« Points », 106), 1979, p. 73.

${ }_{55}^{5}$ L'Österreichischer Rundfunk (Ö 1) diffuse le 11 mai 1970 la lecture de cet extrait.

${ }^{56}$ Cf. la lettre de Benita Bammer au Kurier (11 mai 1970) et celle de Grete Nemec au Wiener Tierschutzverein, dont le vice-président Dr. A. Huber proteste à son tour le 14 mai 1970
} 
de s'interroger sur leur part de conscience et de responsabilité dans l'élaboration et la transmission de la doxa qu'ils imposent à leur progéniture. Les éducateurs ne sont-ils pas tous des éduqués? Et la langue, comme le pense toute une lignée de théologiens, philosophes, théoriciens et écrivains, qui va de saint Augustin aux groupes de Vienne et de Graz, en passant bien sûr par Wittgenstein ${ }^{57}$, ne ment-elle que par l'usage qui en est fait? $\mathrm{Ne}$ faudrait-il pas par exemple confronter l'optimisme professé à cet égard par H. Weinrich dans Linguistik der Lüge ${ }^{58}$ aux réserves émises par Friedrich Kainz dans Über die Sprachverführung des Denkens ${ }^{59}$ ?

L'intentionnalité du mensonge est un critère de définition dont le maintien, ainsi que le remarque Jochen Mecke dans son essai «Lüge und Literatur. Perspektivenwechsel und Wechselperspektive $»^{60}$, resserre par trop l'acception du terme, mais dont l'abandon l'élargit à l'excès. Qu'on le conserve ou l'élimine, qu'on fasse des tournures usuelles un mensonge ou non, délibéré ou pas, il convient en tout cas de nuancer les fondements de la bataille engagée par les auteurs de Forum Stadtpark contre phrases toutes faites et formules figées. L'on se doit aussi d'observer qu'elle débouche sur l'aporie d'un langage dynamité, inutilisable pour communiquer. Les écrivains de Graz sortiront de cette impasse, chacun à sa manière ${ }^{61}$. Ainsi que le note G. Melzer, B. Frischmuth demeurera fidèle à l'enfance, comprise non comme tranche initiale de la vie humaine, mais comme force d'ébranlement de la chose établie, fluidité qui métamorphose à l'infini une réalité donnée pour intangible ${ }^{62}$. Renonçant à l'aura avant-gardiste qui nimbe les jeunes auteurs

auprès de la direction des programmes de l’ÖRF (Franz-Nabl-Institut für Literaturforschung, « Schwarzbauer »-Zeitungsausschnittssammlung).

${ }^{57}$ Cf. les paragraphes 241 et 249 des Philosophische Untersuchungen : " "So sagst du also, daß die Übereinstimmung der Menschen entscheide, was richtig und was falsch ist ?" - Richtig und falsch ist, was die Menschen sagen; und in der Sprache stimmen die Menschen überein. Dies ist keine Übereinstimmung der Meinungen, sondern der Lebensform »; " (Das Lügen ist ein Sprachspiel, das gelernt sein will, wie jedes andre.) »(Wittgenstein, Philosophische Untersuchungen, p. 358 et 356, c'est Wittgenstein qui souligne).

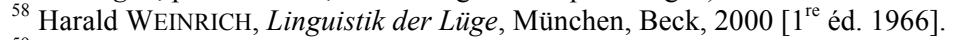
1972.

${ }^{59}$ Friedrich KaINZ, Über die Sprachverführung des Denkens, Berlin, Duncker \& Humblot,

${ }^{60}$ Jochen MECKE, « Lüge und Literatur. Perspektivenwechsel und Wechselperspektive », in Jörn MÜLlER, Hanns-Gregor NISSING (dir.), Die Lüge. Ein Alltagsphänomen aus wissenschaftlicher Sicht, Darmstadt, Wissenschaftliche Buchgesellschaft, 2007, p. 67.

${ }^{61}$ Pour Peter Handke, par exemple, le tournant se situe en 1972 avec Wunschloses Unglück, texte dans lequel il tente d'écrire sur sa mère qui vient de se suicider (cf. Wendelin SCHMIDTDENGLER, Bruchlinien, Salzburg/ Wien, Residenz, 1995, p. 254-268).

${ }_{62}$ « $[\mathrm{S}] \mathrm{o}$ geht es $[\ldots]$ darum, dem Prinzip der Verfestigungen und verhärteten Geltungsansprüche einen Zustand permanenter Verwandlung entgegenzusetzen. Frischmuth faßt diese Polarität als Gegensatz von Herrschaft und Kindschaft [...] : nicht den biologischen Lebensabschnitt will sie festhalten, sondern die Offenheit und Wandlungsfähigkeit, die ihn kennzeichnet» (Gerhard MELzER, «Die Flügel der Kinder. Herrschaft und Kindschaft bei Barbara Frischmuth ", in Daniela BARTENS, Ingrid SPÖRK (dir.), Barbara Frischmuth. Fremdgänge. Ein illustrierter Streifzug durch einen literarischen Kosmos, Salzburg/ Wien..., Residenz, 2001, p. 130, c'est G. Melzer qui souligne). 
de Forum Stadtpark, B. Frischmuth renouera dans les années soixante-dix avec le récit et avec des modes d'écriture inscrits dans une tradition, tel le merveilleux. "Raconter des histoires"... Élargir l'éventail des possibles... N'est-ce pas, au fond, la plus belle preuve de respect envers le réel que la littérature puisse donner? 